\title{
Leaky-Wave Antennas With Arbitrary Radiation Based on Bianisotropic Huygens' Metasurfaces
}

\author{
E. Abdo-Sánchez*(1) ${ }^{(1)}$ A. Epstein ${ }^{(2)}$, and G. V. Eleftheriades ${ }^{(3)}$
}

(1) Dpto. Ing. Comunicaciones, E.T.S.I. Telecomunicación, Universidad de Málaga, Andalucía Tech, E-29071 Málaga, Spain.

(2) Andrew and Erna Viterbi Faculty of Electrical Engineering, Technion - Israel Institute of Technology, Haifa 32000, Israel.

(3) The Edward S. Rogers Sr. Department of Electrical and Computer Engineering, University of Toronto, Toronto, Canada.

\begin{abstract}
In this contribution, we present the application of Huygens' metasurfaces (HMS) to the arbitrary design of leaky-wave antennas (LWAs). These metasurfaces have demonstrated their capabiity to tailor the electromagnetic waves at will. We use them to design LWAs consisting on a parallel-plate waveguide in which the top cover is replaced by a HMS, which implements the desired field transformation. By introducing bianisotropy of the omega type into the metasurface, we have the required degrees of freedom to achieve arbitrary control of the LWA radiation parameters: leakage factor and pointing angle (phase constant). The theoretical derivation of the concept and the design methodology will be described. Moreover, the flexibility in the design will be highlighted with results of two examples of broadside radiation with different directivities.
\end{abstract}

\section{Introduction}

Leaky-wave antennas are guiding structures that leak power along their length. Their radiation is mainly characterized by the propagation constant (leakage factor and phase constant) of the traveling wave. In the recent years, they have received increasing attention due to the demand for lowcost directive antennas with beam steering. Unlike phased arrays, LWAs have a very simple feeding and intrinsic frequency scanning.

On the other hand, metasurfaces are the $2 \mathrm{D}$ version of metamaterials and have been used to manipulate electromagnetic waves at will. Specifically, Huygens' metasurfaces consist of subwavelength electrically- and magneticallypolarizable particles, which are engineered to allow the fulfillment of the required boundary conditions, so that the desired field transformation is achieved. It has been recently discovered that by employing omega-type bianisotropic metasurfaces (O-BMSs) just one condition in the stipulation of the fields must be met to achieve arbitrary field transformation using passive and lossless particles: local power conservation along the metasurface [1]. This is possible due to the additional (magnetoelectric) degree of freedom provided by the O-BMSs.

In this contribution, we propose the combination of a bianisotropic Huygens' metasurface with a guiding structure to

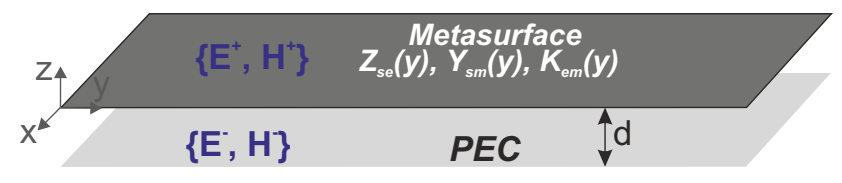

Figure 1. Schematic of the proposed LWA.

build a LWA in which we can engineer the propagation constant to have control on the radiation characteristics. In this way, by placing a Huygens' metasurface on the top of the guiding structure (a parallel-plate waveguide in this case), the required boundary conditions to transform the guiding mode into the desired leaky mode can be implemented.

\section{Theory}

Let us assume a transverse electric (TE) polarized field as excitation $\left(E_{y}=E_{z}=H_{x}=0\right)$ in a parallel-plate waveguide with the top plate being a bianisotropic Huygens' metasurface, as indicated by Fig. 1. Then, the transverse field components above $\left(E_{x}^{+}\right.$and $\left.H_{y}^{+}\right)$and below $\left(E_{x}^{-}\right.$and $\left.H_{y}^{-}\right)$the O-BMS are related through the bianisotropic sheet transition conditions [2]:

$$
\begin{aligned}
\frac{1}{2}\left(E_{x}^{+}+E_{x}^{-}\right) & =-Z_{s e}\left(H_{y}^{+}-H_{y}^{-}\right)-K_{e m}\left(E_{x}^{+}-E_{x}^{-}\right) \\
\frac{1}{2}\left(H_{y}^{+}+H_{y}^{-}\right) & =-Y_{s m}\left(E_{x}^{+}-E_{x}^{-}\right)+K_{e m}\left(H_{y}^{+}-H_{y}^{-}\right)
\end{aligned}
$$

where $Z_{s e}$ stands for the electric surface impedance, $Y_{s m}$ for the magnetic surface admittance and $K_{e m}$ for the magnetoelectric coupling coefficient.

It was demonstrated that a passive and lossless O-BMS, i.e. with $\operatorname{Re}\left[Z_{s e}\right]=\operatorname{Re}\left[Y_{s m}\right]=\operatorname{Im}\left[K_{e m}\right]=0$, can provide a given (arbitrary) field transformation if the local power conservation condition is fulfilled: $P_{z}^{-}(y)=P_{z}^{+}(y)$ [1]. From (1), we can calculate the metasurface parameters $\left\{K_{e m}, Y_{s m}, Z_{s e}\right\}$ to achieve a certain field transformation as follows [1]:

$$
\begin{aligned}
K_{e m} & =\frac{1}{2} \frac{\operatorname{Re}\left[E_{x}^{+} H_{y}^{-*}-E_{x}^{-} H_{y}^{+*}\right]}{\operatorname{Re}\left[\left(E_{x}^{+}-E_{x}^{-}\right)\left(H_{y}^{+}-H_{y}^{-}\right)^{*}\right]} \\
Y_{s m} & =-j\left(\frac{1}{2} \operatorname{Im}\left[\frac{H_{y}^{+}+H_{y}^{-}}{E_{x}^{+}-E_{x}^{-}}\right]-K_{e m} \operatorname{Im}\left[\frac{H_{y}^{+}-H_{y}^{-}}{E_{x}^{+}-E_{x}^{-}}\right]\right) \\
Z_{s e} & =-j\left(\frac{1}{2} \operatorname{Im}\left[\frac{E_{x}^{+}+E_{x}^{-}}{H_{y}^{+}-H_{y}^{-}}\right]+K_{e m} \operatorname{Im}\left[\frac{E_{x}^{+}-E_{x}^{-}}{H_{y}^{+}-H_{y}^{-}}\right]\right) .
\end{aligned}
$$


Then, to be able to design a metasurface that transforms the wave being propagated between the parallel plates into a leaky mode, we firstly need to stipulate the electromagnetic field inside the waveguide that fulfills Maxwell's equations. Since the metasurface design will force the boundary conditions at $z=0$ to be met, the only restriction for the field below the O-BMS is to vanish at the PEC $(z=-d)$. For the region above the metasurface, we desire a leaky mode. Therefore, the following electric fields have been stipulated [3]:

$$
E_{x}^{-}=\left|E_{i n}\right|\left(e^{j k_{z}^{-}(z+d)}-e^{-j k_{z}^{-}(z+d)}\right) e^{-j k_{y}^{-} y}
$$

and

$$
E_{x}^{+}=\left|E_{\text {out }}\right| e^{-j k_{z}^{+} z} e^{-j k_{y}^{+} y},
$$

where the propagation constants are complex:

$$
\begin{aligned}
& k_{y}^{-}=\beta^{-}-j \alpha ; k_{z}^{-}=\beta_{z}^{-}-j \alpha_{z}^{-} ; k^{-2}=k_{y}^{-2}+k_{z}^{-2} \\
& k_{y}^{+}=\beta^{+}-j \alpha ; k_{z}^{+}=\beta_{z}^{+}-j \alpha_{z}^{+} ; k^{+^{2}}=k_{y}^{+2}+k_{z}^{+2} .
\end{aligned}
$$

As can be noticed, the field has the same leakage factor $(\alpha)$ above and below the metasurface to guarantee the power conservation condition. Moreover, the constant $\left|E_{\text {out }}\right|$ is set by the rest of the parameters [3]. However, any more restriction in the choice of the propagation constant of the leaky mode is imposed, thus allowing the independent choice of $\alpha$ and $\beta^{+}$.

\section{Design Procedure and Proof of Concept}

The propagation constant of the leaky mode $k_{y}^{+}$is directly related to the pointing angle of radiation $\theta_{\text {out }}$ and the radiation pattern shape (i.e., directivity, side-lobe level, etc.), since $\beta$ determines the phase distribution and $\alpha$ the amplitude distribution on the antenna. Therefore, we need to design the metasurface for a certain $\beta^{+}$and $\alpha$.

Therefore, we stipulate the fields in (3) and (4) according to the desired radiation characteristics. Additionally, we can set other degrees of freedom, like the waveguide height $d$ or the phase constant inside the waveguide $\beta^{-}$.

Once we have set the fields, we can calculate the metasurface parameters $\left\{K_{e m}, Y_{s m}, Z_{s e}\right\}$ following (2). It can be shown that, for constant $\alpha$, the metasurface parameters $\left\{K_{e m}, Y_{s m}, Z_{s e}\right\}$ result periodic, with a period given by

$$
p=\frac{2 \pi}{\mid \beta^{+}-\beta^{-\mid}} .
$$

This is consistent with Floquet's theory of periodic LWAs.

In order to implement the metasurface that transforms the guided field into the desired leaky-mode, we need to discretize $\left\{K_{e m}, Y_{s m}, Z_{s e}\right\}$ along $y$. For the physical implementation of each unit-cell, three different elements are needed. To realize the O-BMS being compatible with standard fabrication techniques, we use asymmetric three-layer stack of

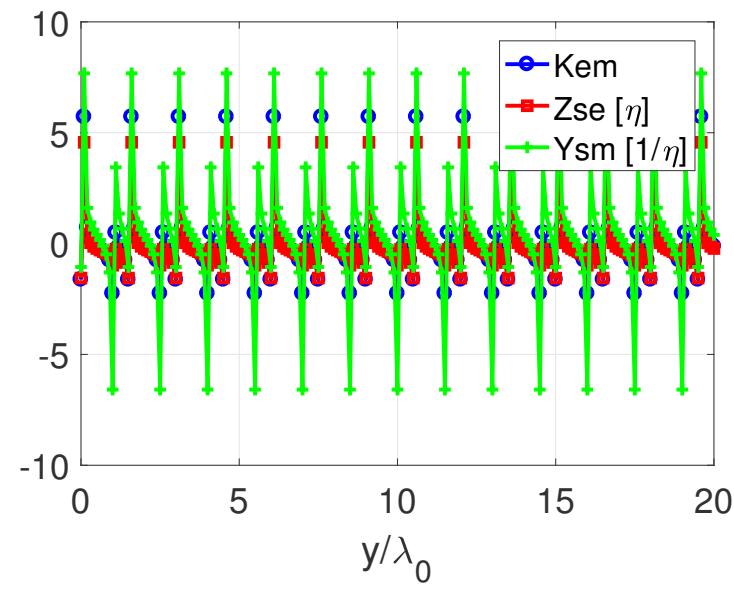

Figure 2. Required metasurface parameters $\left\{K_{e m}, Y_{s m}, Z_{s e}\right\}$ for the design example of $\alpha=0.01 \lambda_{0}$.

impedance sheets, as done in $[1,4]$. In this way, we transform the local $\left\{K_{e m}, Y_{s m}, Z_{s e}\right\}$ into the required Z-matrix for each three-layer unit-cell using (1) and the relations between the tangential fields below and above the metasurface [5]:

$$
\left(\begin{array}{c}
E_{x}^{-} \\
E_{x}^{+}
\end{array}\right)=\left(\begin{array}{ll}
Z_{11} & Z_{12} \\
Z_{21} & Z_{22}
\end{array}\right)\left(\begin{array}{c}
H_{y}^{-} \\
-H_{y}^{+}
\end{array}\right) .
$$

Then, by applying the transmission line model of the threelayer stack of impedance sheets, we transform the matrix $[\mathbf{Z}]$ into the required values of the impedance sheets (which result to be lossless) $\left\{X_{\text {bot }}, X_{\text {mid }}, X_{\text {top }}\right\}$ [1].

As an example, we design a metasurface for broadside radiation for directive aplications. We choose this example since broadside is a traditionally problematic pointing angle for LWAs, due to the open-stopband effect. However, we could radiate at any other pointing angle since we can arbitrarily choose the phase constant $\beta^{+}$[3]. We have chosen a constant leakage factor $\alpha$, therefore the resulting metasurface is periodic. We have arbitrarily set the period $p=1.5 \lambda_{0}$ (which fixes $\beta^{-}$) and the waveguide height $d=0.75 \lambda_{0}$. We have discretized the metasurface parameters with cells $\frac{\lambda_{0}}{10}$-long. In order to get high directivity, we have chosen a low $\alpha\left(0.01 k_{0}\right)$ and a total length of the metasurface $L=20 \lambda$, so that around $90 \%$ of the incident power is radiated. Fig. 2 shows the resulting metasurface parameters that we must synthesize to achieve the desired field transformation and Fig. 3 plots the corresponding reactance sheets for the three layers implementing the metasurface.

In order to prove the concept, we simulate the proposed LWA in the electromagnetic simulator ANSYS HFSS. To simulate the reactance sheets, we use impedance boundary conditions. The resulting 2D directivity is shown in Fig. 4. Very good agreement is found between simulation and the analytical solution. The discrepancies are likely due to the discretization process of the metasurface and the poor convergence of the simulator (due to memory limitations). 


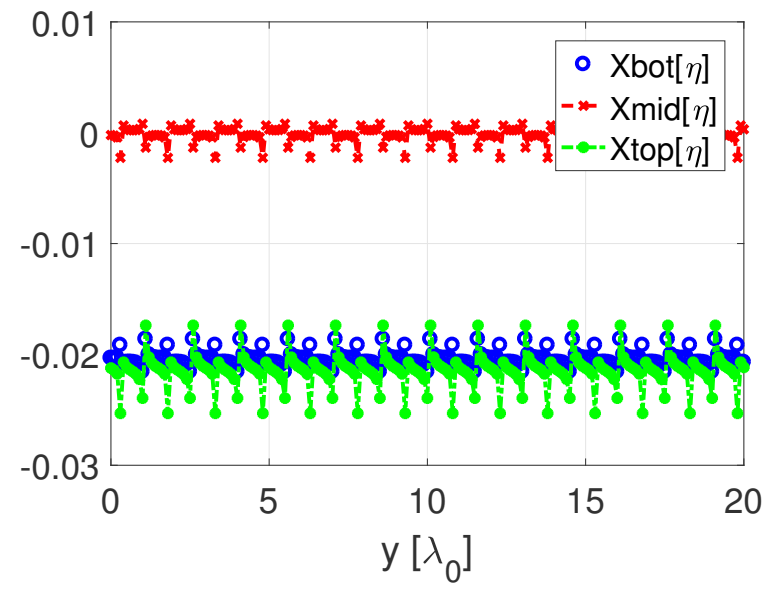

Figure 3. Required reactance sheet values $\left\{X_{\text {bot }}, X_{\text {mid }}, X_{\text {top }}\right\}$ for the design example of $\alpha=0.01 \lambda_{0}$.

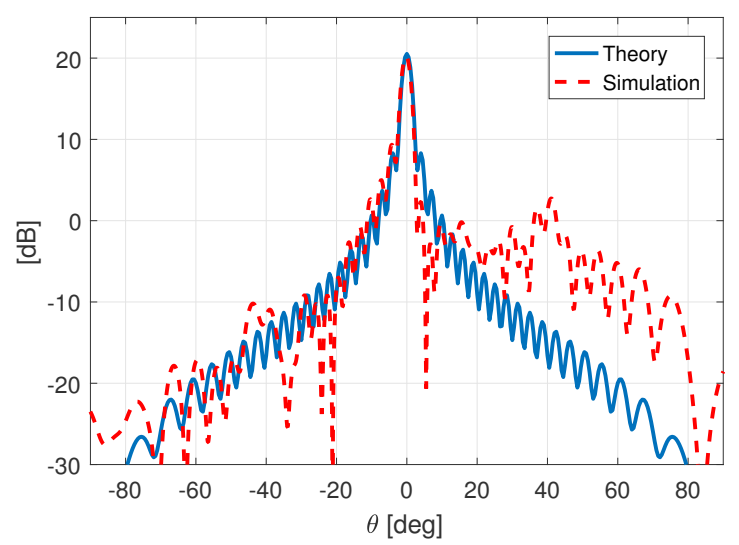

Figure 4. 2D directivity comparison for the design example of $\alpha=0.01 \lambda_{0}$.

Instead of directive radiation, wide beam can be useful for some applications. We can design a LWA with wide broadside beam by choosing a higher leakage factor and a shorter antenna. With this purpose, we have fixed $\alpha=0.04 k_{0}$ and $L=5 \lambda_{0}$, while keeping the rest of the parameters the same as in the previous example. Fig. 5 shows the resulting directivity. Since this structure is shorter, the truncation effects will be more pronounced and more discrepancies between the analytical solution and the simulation are expected. However, good agreement is found in the main lobe. It is highlighted that the freedom in the choice of the propagation constant of the leaky mode leads to potential flexibility in the radiation pattern.

A step further would be the realization of the metasurface with a physical structure. To emulate the required reactances, we would need elements that cover both inductive and capacitive behavior. An implementation possibility for the unit-cell is the use of three stacked copper layers with dogbone shape, as in [1]. The final antenna performance will then depend on how close the reactance values can be synthesized with physical unit-cells. Moreover, other ef-

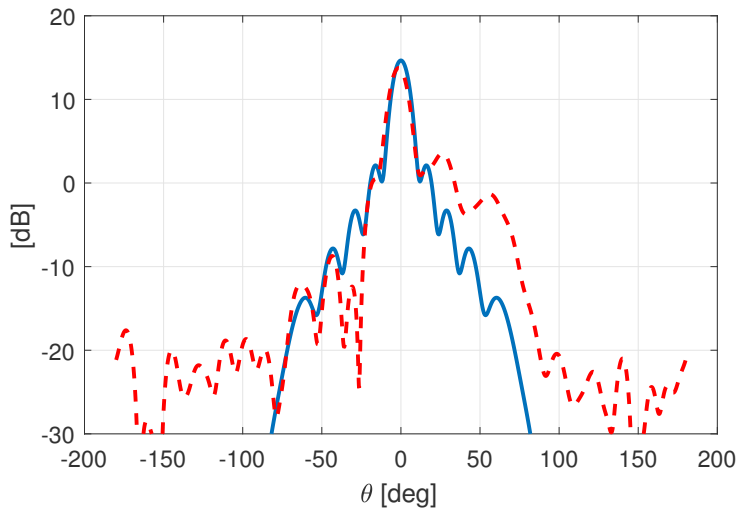

Figure 5. 2D directivity comparison for the design example of $\alpha=0.04 \lambda_{0}$.

fects like substrate losses should be taken into account.

\section{Conclusion}

The concept of a LWA based on a parallel-plate waveguide with the top cover being a bianisotropic Huygens' metasurface has been presented. The theoretical derivation has been detailed and the design methodology has been described and illustrated with two examples of broadside radiation with different leakage factors (directive radiation and wide beam). The provided results validate the proposed design methodology to implement LWAs with flexible choice of the radiation parameters. The metasurface guarantees the required boundary conditions to be met so the field above the antenna is the one we have stipulated. Ideally, arbitrary choice of the pointing angle [3] and the leakage factor is possible. Additionally, the waveguide height and the period are also degrees of freedom in the design. Further work will focus on the physical implementation of the metasurface and the modulation of the leakage factor along the structure.

\section{Acknowledgements}

This project has received funding from the European Union's Horizon 2020 research and innovation programme under the Marie Sklodowska-Curie grant agreement No 706334.

\section{References}

[1] A. Epstein and G. V. Eleftheriades, "Arbitrary PowerConserving Field Transformations with Passive Lossless Omega-Type Bianisotropic Metasurfaces," IEEE Trans. Antennas Propag., vol. 64, no. 9, pp. 38803895, Sept. 2016.

[2] Y. Ra'di and S. A. Tretyakov, "Balanced and optimal bianisotropic particles: Maximizing power extracted from electromagnetic fields," New J. Phys., vol. 15, no. 5, p. 053008, 2013. 
[3] E. Abdo-Sánchez, A. Epstein and G. V. Eleftheriades, "Bianisotropic Huygens' Metasurface LeakyWave Antenna with Flexible Design Parameters," European Conference on Antennas and Propagation., Paris, Mar. 2017.

[4] J. P. S. Wong, A. Epstein, and G. V. Eleftheriades, "Reflectionless wide-angle refracting metasurfaces," IEEE Antennas Wireless Propag. Lett., vol. 15, pp. 1293-1296, 2016.

[5] A. Epstein and G. V. Eleftheriades, "Huygens' metasurfaces via the equivalence principle: design and applications," J. Opt. Soc. Am. B, vol. 33, pp. A31-A50, Feb 2016. 